\title{
AN OVERVIEW OF APPLICATIONS OF INDUCTION HEATING
}

\author{
Ketul M Patel \\ Electrical Engineering Department, Dr. S. \& S. S. Ghandhy Government Engineering \\ College, Surat, Majura Gate, Surat, Gujarat, India
}

\begin{abstract}
ABTRACT
Eddy currents are normally associated with losses and heating in electrical equipments and ways to mitigate the same. But this heating can be used productively for various domestic and industrial applications. In this paper, various applications related to induction heating are discussed. Various aspects of heat treatment and metal forming using induction heating have been discussed. Various technological aspects of induction melting process involving induction furnaces for melting of ferrous metals, metals with high purity and silicon have been discussed. Induction heating finds application in food processing and in today's era, widespread application in cooking. So various aspects of induction heating-based food processing and induction cooking have also been discussed.
\end{abstract}

Keywords: Eddy current, Food Processing, Induction Heating, Heat Treatment, Induction Furnace, Induction Melting.

Cite this Article: Ketul M Patel, An Overview of Applications of Induction Heating, International Journal of Electrical Engineering and Technology, 10(2), 2019, pp. 81-85. http://iaeme.com/Home/issue/IJEET?Volume=10\&Issue $=2$

\section{INTRODUCTION}

Whenever a conducting body is subjected to changing magnetic field, emf is induced in that conducting body and currents flow if there exists a closed path. But if this conducting body or the object is large, then such induced emf will make currents to circulate within this conducting body in appropriate directions which are known as eddy currents. The magnitude of the induced emf depends upon the strength of the magnetic field and the coupling of this field with the object. The magnitude of eddy currents is dictated by the induced emf and the conductivity of the object. These eddy currents, when circulating within the object, produce large amount of losses and heating. The heating so produced in the object due to eddy current may be put to effective use for various applications which are broadly known as induction heating. Major advantages of induction heating are efficient, effective and uniform heating, better control of heating process, cleanliness and environment friendliness, better product quality and reduction in heat losses. Induction heating is used in various applications like heat treatment, metal forming, induction melting, food processing, induction cooking etc. 
In heat treatment, the surface of the metal is heated to desired depth using coils surrounding the object which are supplied with power supply of suitable strength and frequency and then rapidly quenched to obtain desired surface characteristics. In metal forming using induction heating, complete work-piece is heated to high temperatures and then pressed, rolled, hammered or extruded to obtain desired thickness, shape and structure of the work-piece. Induction furnaces employ large size coils carrying heavy currents to produce eddy currents and hence heating in the material contained in the crucible to a temperature high enough to obtain melting of the material. Induction cooking has come a long way and has now found wide-spread use in cooking applications where, a coil is placed inside a casing and this induces currents and hence heating the vessel placed above the induction cook-top and consequently food contained in the vessel gets cooked. Induction heating also finds application in food processing industry. Food processing involves drying of food in controlled conditions for which food can be heated using induction heating principle.

\section{HEAT TREATMENT}

Induction heat treatment involves heating of the work-piece to a certain temperature, maintaining temperature at such level for a definite period and then quickly quenching it, and all of these are done in a controlled fashion, thereby obtaining the desired properties of the work-piece. While applying heat treatment to any work-piece, the depth of application of heat is also controlled by using suitable frequency of supply. Heat treatment includes various processes are hardening, tempering, normalizing, annealing spheroidizing and sintering.

The hardening can be broadly subdivided into two categories as surface hardening and through hardening. In surface hardening, the treatment is applied to the surface layer of the work-piece up to certain depth as desired. In through hardening, heat is applied uniformly to the whole mass from surface to the core of the work-piece. The objects like bearings, which normally are required to have wear resistance, require shallow depth of heat treatment and the depth in such parts is controlled between 0.25 and $1 \mathrm{~mm}$, whereas in objects like gears, where both wear resistance and some amount of loading are applicable, depth of hardening is controlled from about 1 to $3 \mathrm{~mm}$. The objects like axle shaft, which are subjected to heavy loading, are hardened for a depth of around 3 to $10 \mathrm{~mm}$. As the depth of heating varies from fraction of $\mathrm{mm}$ to few $\mathrm{mm}$, the frequency varies in the range from few hundred $\mathrm{kHz}$ to few $\mathrm{Hz}$, giving the desired penetration of heating into the surface of the object and the heating time may vary from 1 to 10 seconds for a single work-piece. Appropriate and precisely controlled metallurgical properties of work-piece and properly controlled process parameters like temperature, time and supply frequency are essential for obtaining desired properties in the work-piece. Through hardening is employed when high strength is required. In this type of hardening, the work-piece is uniformly heated from surface to the core of the object. The supply frequencies are much lower in this case as compared to surface hardening to achieve penetration of currents up to the core of the work-piece. Careful selection of frequency for through hardening is essential as otherwise, the depth of current penetration may increase and cancellation of eddy currents may result reducing the effectiveness and efficiency of heating. The process of tempering is carried out after hardening. In this process, the work-piece is reheated after hardening for relaxation or reduction of internal stresses that have got developed in the process of hardening. Tempering relieves the stresses and improves properties of the work-piece and retains, to a great extent, the hardness acquired through hardening process.

\section{METAL FORMING}

In this process, the work-piece is heated to temperature that makes it suitable for forming processes like forging, rolling, extrusion etc. This process can also be used for heating of object during coating operation. The work-piece is uniformly heated to required temperature. As 
uniform heating is required and large mass is involved, such process demands heating with high power and relatively lower frequencies. Once uniformly heated, the work-piece is subjected to a hammer, press or roll former or extrusion machine where desired forming process is carried out and desired thickness/diameter and shape is obtained. Induction heating can also be employed coating, spraying and curing of various paints, varnishes etc. In the process of curing, the material is first applied the desired layer and then is subjected to induction heating. As induction heating gives uniform heating throughout the layer, the quality of coating is superior. In the process of thermal spraying, the material surface is pre-heated with the help of induction coils and then is subjected to spray system for applying desired spray coating.

\section{INDUCTION MELTING}

Induction furnaces have been in use since more than 100 years for melting of iron and steel. The induction furnaces are also used for melting of high purity metals such as Titanium, Niobium, Molybdenum etc. and also for melting of semiconductor silicon in which melting with high control over crystalline structure is essential. The induction furnaces are less prone to chemical reactions. However, the melting process is highly affected by the quality and composition of materials. Induction furnaces are more environment friendly, producing less fumes and smoke.

In conventional combustion furnace, heat is produced by using conventional fuels like coal and natural gas, thereby raising the temperature inside the furnace as much high as is required for melting the material inside the furnace. In case of induction furnaces, the material to be melted is heated directly by the principle of eddy current. The furnace is surrounded by induction coils normally made-up of hollow copper tubes. These coils have to carry heavy current and therefore considerable losses get produced in these coils. Therefore they are needed to be cooled effectively for which, many times, water cooling is used, passing cooling water through the hollow coils. Through these coils, heavy alternating current is passed in controlled manner, which produces magnetic field of desired strength and this field links with the material which is to be melted. This changing magnetic field linking with the material produces eddy currents within the material mass. These currents produce $I^{2} R$ losses inside the material and heat the material. The amount of heating depends on the magnitude of current produced and the resistivity of the material being heated. So in this type of furnace, instead of heating the furnace, the material is heated directly. Induction heating provides inherent stirring action, which helps in obtaining good homogeneity in the material being melted. The melting process becomes fast and more effective when it is done at higher frequencies. When the furnace's heating power supply frequency is increased, the power density of the furnace increases and the melting of the material can be accomplished in lesser time. Normally AC-DC-AC conversion is used for supplying the furnace; i.e., first the AC supply is converted into controlled DC supply, generally using heavy duty SCR based rectifiers and then, using suitable rating inverter, this DC supply is converted back into AC supply of desired frequency and magnitude. Such power conversion produces harmonics in the utility supply which are needed to be controlled. Also induction furnaces suffer from inherent low power factor. So special measures are to be incorporated for purifying the utility supply and maintaining power factor as may be imposed by the utility requirements.

For melting of high purity metals, cold crucible type or float-zone type process is employed. In the cold crucible process, a water cooled metallic crucible is used, in which, when the molten material comes into contact with the crucible, gets solidified immediately, forming a solid crust between the metal and inside surface of crucible. Thus this crust acts as barrier, avoiding direct contact of molten material with the crucible, thereby allowing melting without impurities. This is possible as the heat is transferred through induction process and no direct contact between the material and the furnace crucible is necessary. This process can be used both for 
semiconductor silicon as well as pure metals. The float-zone process is very useful for refining or single crystal growth. The float zone process is used for obtaining refined or single crystal growth from polycrystalline electronic grade silicon (EGS). In this technique, a polycrystalline EGS rod is fused with single crystal seed having desired orientation. An inert gas furnace is used which employs a travelling radio frequency coil. This coil begins at the fused region, travelling towards the polycrystalline EGS rod. The material of this rod gets melted due to the heating provided by this coil and when this molten region solidifies, due to the fusing with the seed, it obtains the same orientation as that of the seed. The purpose of inert gas in the furnace is to avoid any gaseous impurities and to facilitate production of oxygen free silicon.

\section{INDUCTION BASED FOOD PROCESSING AND COOKING}

Food is a non-ferrous material and hence cannot be heated directly through induction heating, i.e. it is not possible to induce the current directly in the food material and obtain heating. Instead, a ferrous material is used as a vessel in which the food is placed.

The food processing industry conventionally has been using steam and hot air for heating the food in the desired manner. But this suffers from low processing efficiency. Induction heating can be a good alternative for food processing. Various methods have been developed over the years for processing food through induction heating. In one method, series of trays with food placed on the tray are arranged one over the other. The tray has a layer of ferrous material facilitating heating through induction and induction coils are inserted in-between the trays. These coils heat the ferrous material layer and get heated and this heat is used for heating the food on the tray. In another technique, a cylindrical metallic vessel is used which rotates on longitudinal axis. Food to be processed is entered at one end of the cylinder, and travelling inside the cylinder, exit at the other end of the cylinder. The induction coils are placed around this cylinder, along its length in a circumferential manner, which induce heat uniformly throughout the wall of the cylinder. This cylinder is thermally insulated above the heating coils to avoid heat losses from the exterior surface of the cylinder. As the food material passes through, this heat of the cylinder walls is transferred to the food material travelling inside the cylinder.

In case of induction cookers, the induction cook-top contains a coil in which, high current at medium high frequency is supplied. This coil produces magnetic field which induces currents in the vessel put on the cook-top. This vessel gets heated due to the losses due to this current and it transfers heat to the food to be cooked. In case of non-ferrous vessel like glass, copper, non-ferrous alloys of stainless steel etc. are required to be used for cooking, a sheet of ferrous material has to be used as an interface which gets heated itself and then transfers the heat to the non-ferrous vessel. Generally a planar coil is used for producing the desired magnetic field. Desired amount of heating is obtained in the vessels by suitably changing the magnetic field and thereby controlling the currents that are induced in the vessel or the interface, thereby controlling the heat produced. To achieve this control, the coil is generally fed by power electronics inverter. Generally, a multi-turn coil is housed in suitable housing below the vessel and electric power with frequencies in the range of few tens to few hundred $\mathrm{kHz}$. Induction cook-tops give higher efficiency, safety, cleanliness and more advanced and effective control over the cooking process.

\section{CONCLUSION}

Induction heating finds wide applications in industrial as well as domestic environment. Induction heating gives efficient, effective and uniform heating, better control of heating process, cleanliness and environment friendliness, better product quality and reduction in heat losses. Heat treatment is an area in which induction heating is extensively used and provides great deal of control and very good control over properties and quality of product. Induction 
melting is also very popular in industrial environment and gives an impurity free and homogeneous melting with efficient heating. Various aspects of heat treatment, metal forming, induction forming, food processing and cooking from the perspective of induction heating principle have been discussed in this paper. Various advantages of induction heating over other conventional processes in these areas have been highlighted.

\section{REFERENCES}

[1] Oscar Lucía, Pascal Maussion, Enrique J. Dede et al., Induction Heating Technology and Its Applications: Past Developments, Current Technology, and Future Challenges, IEEE Transactions on Industrial Electronics, Vol. 61, No. 5, May 2014

[2] Valery Rudnev, Don Loveless, Raymond Cook et al., Handbook of Induction Heating, 5, 8, (Marcel Dekker Inc 2003)

[3] A. Mühlbauer, Innovative Induction Melting Technologies: A Historical Review, International Scientific Colloquium: Modelling for Material Processing, Riga, June 8-9, 2006, 13-20

[4] Oscar Lucia, Jesus Acero, Claudio Carretero et al., Induction Heating Appliances: Toward More Flexible Cooking Surfaces, EEE industrial electronics magazine, Sept 2013, 35-47.

[5] J. Acero, J.M. Burdio, L.A. Barragán et al., The domestic induction heating appliance: an overview of recent research, IEEE Applied Power Electronics Conference and Exposition 2008,651-657

[6] ivek R. Gandhewar, Satish V. Bansod and Atul B.Borade, Induction Furnace - A Review, International Journal of Engineering and Technology Vol.3 (4), 2011, 277-284

[7] Hamed M. El-Mashad, Zhongli Pan, Application of Induction Heating in Food Processing and Cooking, Food Engineering Reviews June 2017, Volume 9, Issue 2, pp 82-90 